\title{
Particulate gravity currents along V-shaped valleys
}

\author{
JOE J. MONAGHAN ${ }^{1} \dagger$, CATHERINE MÉRIEUX ${ }^{1}$, \\ HERBERT E. HUPPER T ${ }^{2}$ AND JOHN MANSOUR ${ }^{1}$ \\ ${ }^{1}$ School of Mathematical Sciences, Monash University, Vic 3800, Australia \\ ${ }^{2}$ Institute of Theoretical Geophysics, Department of Applied Mathematics and Theoretical Physics, \\ University of Cambridge, CMS, Wilberforce Road, Cambridge CB3 0WA, UK
}

(Received 11 August 2008 and in revised form 16 March 2009)

This paper extends previous studies of saline gravity currents at high Reynolds number flowing along a tank with a V-shaped valley. We use experiments and a box model to determine the primary features of the flow. The particulate gravity currents were initiated by releasing a fixed volume of fluid consisting of pure water mixed with silicon carbide particles from a lock at one end of the tank. The resulting motion and deposit pattern differ significantly from those for the propagation of a particulate gravity current along a flat-bottomed tank. The front of the current, seen from above, is approximately parabolic (with axis parallel to the flow direction) in contrast to the current in a flat-bottomed tank where it is nearly a straight line perpendicular to the flow. This feature mimics the results for pure saline currents. When seen in profile the currents do not have a clearly defined raised head, which is a feature of the flat-bottomed currents. The mass deposited per unit area varies nearly monotonically with respect to distance down the tank, again in contrast to the case of the flat-bottomed tank. The exceptions to this are the two experiments which have the highest ratio of lock height to length. The mass deposited per unit area across the V-shaped valley is much larger in the central part of the valley than it is on the flanks for any position along the valley. We find that the results can be described with remarkable accuracy by a box model using a generalization of the equation for sedimentation from a turbulent medium due to Martin and Nokes. Our results further show that the factor used in the deposition rate equation which is commonly assumed to be 1 should be smaller, typically 0.7 .

\section{Introduction}

Gravity currents in nature usually flow over complex terrain which can include valleys, basins, changes of slope and obstacles which may completely or partially block the flow (for a convenient and comprehensive discussion of field observations of gravity currents, and the associated experimental work, the review of Kneller \& Buckee (2000) is extremely useful). Most experimental work has focused on gravity currents flowing in tanks designed so that the flow is close to two-dimensional. The simplest of these tanks are flat bottomed (Simpson 1997 provides a comprehensive description of experiments using these tanks). The effect of obstacles in the form of ridges on the flow and deposit of particulate matter has also been studied (Rottman,

$\dagger$ Email address for correspondence: joe.monaghan@sci.monash.edu.au 
Simpson \& Hunt 1985; Rottman \& Simpson 1989; see also Kneller \& Buckee 2000 for further references). A related problem which has been studied experimentally is the flow of gravity currents down ramps onto a horizontal surface (Britter \& Linden 1980; Monaghan et al. 1999).

There has been much less research on gravity currents flowing along valleys. Similar solutions for low Reynolds number currents in V-shaped valleys have been determined by Takagi \& Huppert $(2007,2008)$, and shown to accurately predict the experimental results while high-Reynolds-number flows of saline currents along V-shaped valleys have also been studied both theoretically and experimentally (Monaghan et al. 2008). The results show that the change in topography causes significant changes in most properties of a gravity current. For example, in the case of saline currents flowing at high Reynolds number, the currents in the V-shaped valley, when seen in profile, no longer have the raised head characteristic of the flat-bottomed case (see, for example, figure 2 of Rottman \& Simpson 1983). When seen from above, the front has a parabolic shape. In addition, the variation of the speed of the head with time is different from that in the case of a flat-bottomed valley.

Experiments involving gravity currents carrying particulate matter provide useful models of pyroclastic and turbidity flows (comprehensive discussions of the occurrence of particulate-driven gravity currents are given by Huppert 1998 and by Kneller \& Buckee 2000). During the flow, which is usually highly turbulent, the particulate matter is deposited on the valley bottom and, as a consequence, the density difference between the current and the ambient fluid is reduced and the flow slows down. Studies of such currents flowing in horizontal tanks with flat bottoms have been carried out by Bonnecaze, Huppert \& Lister (1993) for constant volume currents, and by Garcia (1994) and Altinakar, Graf \& Hopfinger (1996) for constant flux currents. Bonnecaze et al. (1993) compared their experiments with calculations based on a two-layer fluid model together with an equation due to Martin \& Nokes $(1988,1989)$ which describes the loss of particulate matter in a turbulent flow. The agreement between the experimental and numerical results for the variation of the length of the current with time was, however, very good. The agreement between the variation of the mass deposit with distance for their numerical model and the experiments was satisfactory except in the early stages of the flow where the experiments indicate a significantly lower deposit than the theoretical models. Dade \& Huppert (1995a) presented an approximate box model based on a first approximation for which the deposit did not affect the motion of the front, and this gives a reasonable fit to the experiments.

The only previous experiments of particulate carrying gravity currents flowing along valleys are those of Mohrig \& Buttles (2007) who studied the deposition of particulate matter flowing in a tank with a bed of silica particles in which was carved a shallow channel with two bends. Their experiments show that a channel can have a significant effect on the deposit even when the height of the current is a factor of four greater than the depth of the channel. In contrast with Mohrig \& Buttles (2007), this paper considers channelized currents flowing along a non-erodible valley that defines the geometry of the tank. As a consequence, the lateral spreading of the currents and possible flow over the channel margins, and the erosion of a pre-existing channel that occurred in the experiments of Mohrig \& Buttles (2007) do not occur in our experiments.

The key questions answered in this paper are first, the extent to which the topography of the V-shaped valley affects the deposit pattern in the valley and second, how it affects the speed of the current. We first describe currents of constant volumes in the flat-bottomed case and compare data from both Bonnecaze et al. (1993) 
and our own experiments with results from our box model to provide a reference for our V-shaped-valley experiments for fixed volumes. We then describe the currents in the V-shaped valley. The experiments reveal that the deposit pattern is strikingly different from that in the flat-bottomed tank. In particular, the mass deposited varies nearly monotonically with respect to distance down the tank, in contrast to the case of the flat-bottomed tank. The deposit across the $\mathrm{V}$-shaped valley, for any position along the valley, is much larger in the central part of the valley compared to the flanks. We find that the results can be described with remarkable accuracy by a box model which uses a generalization of the mass deposit equation of Martin \& Nokes $(1988,1989)$ to take account of the geometry of the valley. The experiments are described in $\S 2$. In $\S 3$, we present the flow patterns, which we observed in both the experiments with the flat- and V-shaped-bottom tank. Section 4 details our box model and experiments for the flat-bottomed tank. Section 5 describes the box model and experiments for the $\mathrm{V}$-shaped valley. We discuss our results and concludes in $\S 6$. The sensitivity of the Froude number and a sedimentation parameter in our box models is discussed in the Appendix.

\section{The Experiments}

\subsection{The experimental setup}

The experiments were carried out in a rectangular tank made of Perspex with two sets of floors (figure 1). The primary floor had a V-shaped bottom while the second floor was flat and could be inserted along the tank at the height of the valley $a$. Dimensions of the tank were averaged from four sets of measurements along the tank because, subsequent to the design of the V-shaped-bottom tank, we found that lengths could vary by $2-3 \mathrm{~mm}$. The tank was $500 \mathrm{~cm}$ long with a depth of $40 \mathrm{~cm}$ with the flat bottom, and an averaged maximum depth of $46.6 \mathrm{~cm}$ for the V-shaped bottom, corresponding to a valley of depth $a=6.6 \mathrm{~cm}$. The averaged width of the tank $W$ was $28.26 \mathrm{~cm}$. Note that the slope of the valley walls was then inclined at $25^{\circ}$ to the horizontal, which is an angle less than the typical values of the angle of repose for angular materials, i.e. $30^{\circ}-40^{\circ}$. This implied that no movement of the settled particles was to occur after deposition. We note that our deposits never showed any ripples or irregularities at the surface that could have suggested sliding and/or re-suspension.

The tank was horizontal and initially filled with tap water up to a fixed depth $h_{0}$ measured from the surface of the water to the bottom of the valley. A removable lock gate with foam seals around its edges was positioned at a fixed distance $L=13 \mathrm{~cm}$ from one end of the tank. The characteristic diameters of the silcone carbide (SiC) particles were in the range 20-32 microns and we used the mid-point value 26 microns for our models. A measured mass $m_{0}$ of particles composed of $\mathrm{SiC}$ was added to the volume of water held behind the lock gate. This mixture was stirred vigorously to bring all the particles into suspension before the gate was rapidly lifted. Each experiment was recorded by a video camera.

Measurements of the position of the front of the current as a function of time were obtained from frame by frame replay of the video recording. Measurements of the deposits were made once the particles had settled and the tank had been carefully drained.

Strips of fixed length within a range $5-10 \mathrm{~cm}$ were then cut into the wet cohesive deposits across the width of the tank at given distances along the tank. In two of the V-shaped-valley experiments ( 3 and 5, table 1), each strip was cut into three slices consisting of two symmetric flank sub-strips, each of approximate length $12 \mathrm{~cm}$, and a 


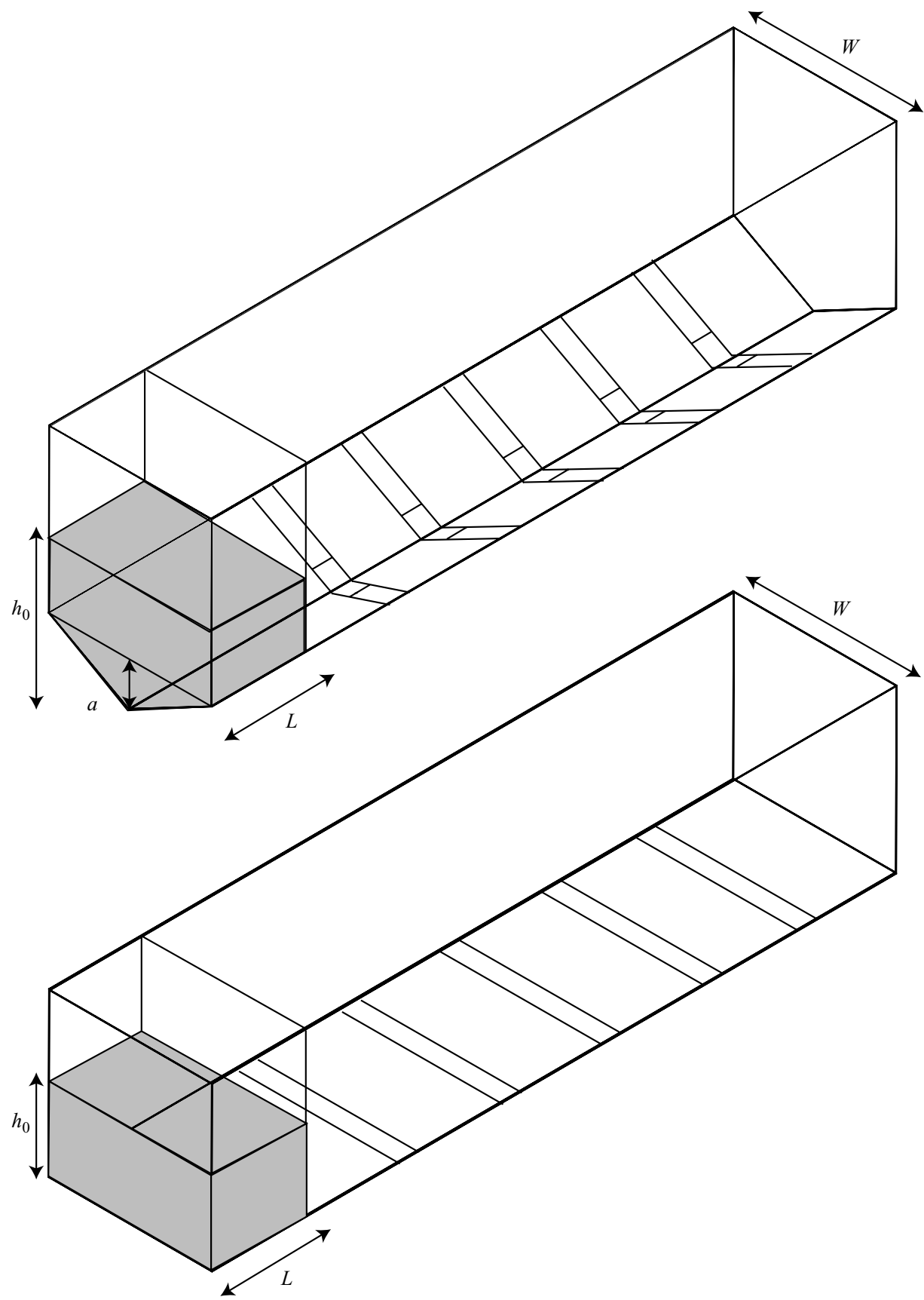

FIGURE 1. Geometry of the tanks: (a) V-shaped valley and (b) flat-bottomed tank. The evenly spaced strips from which mass samples were taken are indicated.

central sub-strip of approximate length $6 \mathrm{~cm}$ (see figure 1). The mass of the particles within each strip was collected in a beaker, then dried in a microwave oven and weighed. The mass of particles that had remained in the lock were also collected and weighed. This mass ranged from $5 \%$ to $10 \%$ of the initial mass. 


\begin{tabular}{|c|c|c|c|c|c|c|c|c|}
\hline Experiment & Valley & $\begin{array}{c}\left(\rho_{c}-\rho_{a}\right) / \rho_{a} \\
(\%)\end{array}$ & $\begin{array}{l}h_{0} \\
(\mathrm{~m})\end{array}$ & $\begin{array}{l}m_{0} \\
(\mathrm{~kg})\end{array}$ & $\varphi_{0}$ & $\begin{array}{c}g_{0}^{\prime} \\
\left(\mathrm{m} \mathrm{s}^{-2}\right)\end{array}$ & $\gamma$ & $R e$ \\
\hline 1 & Flat & 4.6 & 0.049 & 0.123 & 0.021 & 0.45 & 0.7 & 7276 \\
\hline 2 & Flat & 4.6 & 0.115 & 0.289 & 0.023 & 0.45 & 0.5 & 26161 \\
\hline 3 & V-shape & 4.5 & 0.066 & 0.082 & 0.021 & 0.45 & 0.7 & 11374 \\
\hline 4 & V-shape & 4.8 & 0.126 & 0.246 & 0.022 & 0.48 & 0.7 & 30987 \\
\hline 5 & V-shape & 4.4 & 0.126 & 0.225 & 0.021 & 0.44 & 0.7 & 29668 \\
\hline 6 & V-shape & 6.1 & 0.126 & 0.310 & 0.028 & 0.60 & 0.7 & 34644 \\
\hline 7 & V-shape & 2.3 & 0.246 & 0.268 & 0.011 & 0.23 & 0.5 & 58515 \\
\hline 8 & V-shape & 2.3 & 0.126 & 0.117 & 0.011 & 0.23 & 0.7 & 21450 \\
\hline 9 & V-shape & 1.0 & 0.246 & 0.114 & 0.004 & 0.10 & 0.7 & 38584 \\
\hline 10 & V-shape & 1.0 & 0.126 & 0.050 & 0.004 & 0.10 & 0.7 & 14143 \\
\hline
\end{tabular}

TABLE 1. Initial experimental conditions. The density of the particle material $\rho_{p}$ is $3190 \mathrm{~kg} \mathrm{~m}^{-3}$, the density of water $\rho_{a}$ is $\sim 1000 \mathrm{~kg} \mathrm{~m}^{-3}$. The mean diameter of the SiC particles was $26 \mu \mathrm{m}$. $\gamma$ refers to the factor introduced and discussed in (4.1). The initial Reynolds number is estimated by using $R e=\sqrt{g_{0}^{\prime} h_{0}^{3} / \nu^{2}}$, where the kinematic viscosity of water $v$ is $10^{-6} \mathrm{~m}^{2} \mathrm{~s}^{-1}$.

The currents are driven by the effective gravity

$$
g^{\prime}=\left(\rho_{c}-\rho_{a}\right) g / \rho_{a},
$$

where $\rho_{c}$ is the density of the current, $\rho_{a}$ is the density of water and $g$ is the gravitational acceleration. Equation (2.1) can be written in terms of the mass $m$ of the particulate matter in the current at any time and the mass $M$ of the water in the lock and the densities, which give

$$
\rho_{c}=(m+M) /\left(m / \rho_{p}+M / \rho_{a}\right),
$$

where $\rho_{p}$ is the density of the particles.

Substituting for $\rho_{c}$ in (2.1) gives

$$
g^{\prime}=g \frac{m}{M} \frac{\left(1-\rho_{a} / \rho_{p}\right)}{\left(1+m \rho_{a} / M \rho_{p}\right)} .
$$

In this paper the volume concentrations of particles in the current

$$
\varphi=\frac{m}{M} \frac{\rho_{a}}{\rho_{p}}
$$

are assumed to be small. Values of $\varphi$ in the experiments are much less than unity with a typical value of 0.03 . Thus, to within an error of $3 \%,(2.3)$ can be approximated by

$$
g^{\prime}=g\left(1-\rho_{a} / \rho_{p}\right) \frac{m}{M} .
$$

Table 1 gives the parameters of each experiment, where the initial values of $h, m, \varphi$ and $g^{\prime}$ are denoted by $h_{0}, m_{0}, \varphi_{0}$ and $g_{0}^{\prime}$, respectively. Table 1 also includes the factor $\gamma$ that is discussed in $\S 4.1$ and the initial Reynolds numbers of each experiment, which shows that the gravity currents were turbulent.

\section{The flow patterns}

In this section we compare general features of the flow along a flat-bottomed tank and the flow along a V-shaped valley. Figure 2 shows the gravity currents seen from 
(a)

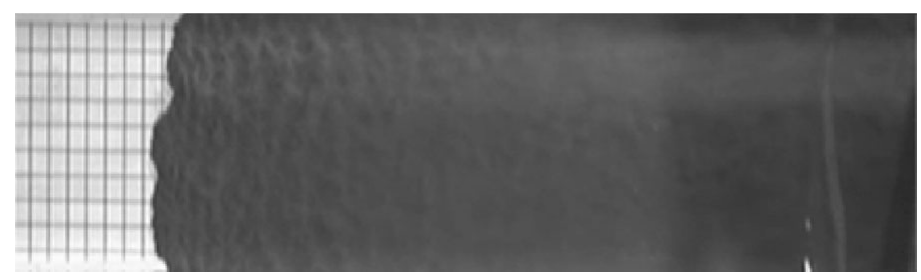

(b)

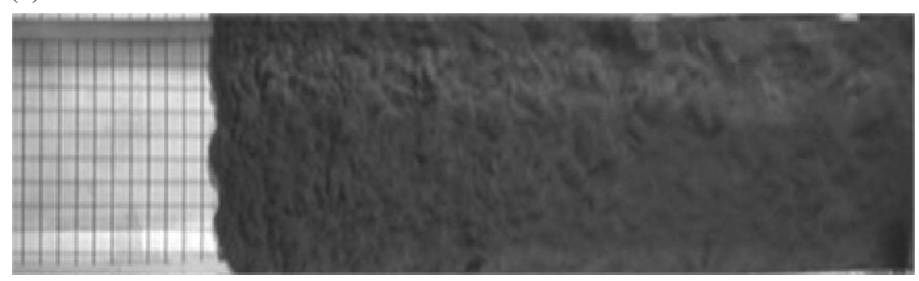

FIGURE 2. Particulate gravity currents from above flowing in a flat-bottomed tank: $(a)$ and (b) correspond to experiments 1 and 2 of table 1 . Note the nearly straight front and turbulent structures near the surface roughly parallel to the front in $(a)$, and the weak turbulence away from the head of the current especially in $(a)$.

(a)

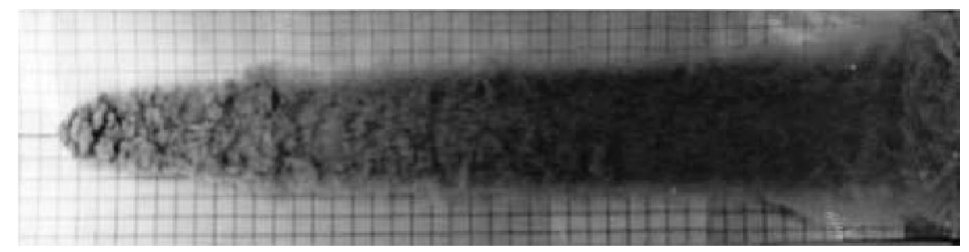

(b)

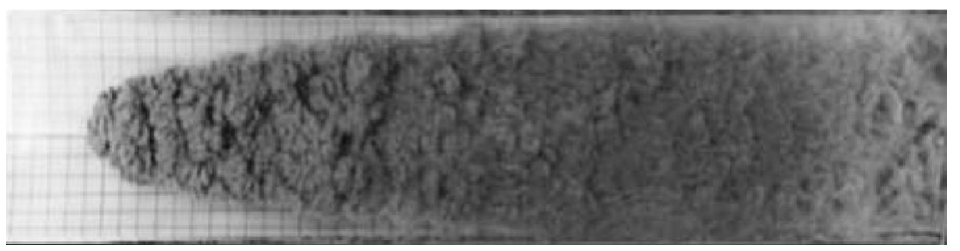

FIGURE 3. Particulate gravity currents from above flowing along a V-shaped valley: $(a)$ and (b) correspond to experiments 3 and 4 of table 1 . Note the shape of the front and the fact that the turbulence remains strong along most of the current.

above for experiments 1 and 2 (flat bottom). The flows are close to two-dimensional with fronts which show small convolutions about a straight line. In both figure 2(a) and $2(b)$ it appears that the turbulence is strong near the head but decreases rapidly with distance from the head. In figure $2(a)$ the structure in the turbulence seen from above appears to be aligned parallel to the front, but this is less clear in figure $2(b)$. A qualification we need to make to these comments is that because the current fluid is opaque we are constrained to surface observations.

Figure 3 shows the gravity currents seen from above for experiments 3 and 4 (V-shaped valley). In this case, as with saline gravity currents, the front of the current is parabolic with a smaller radius of curvature for the current with the smaller value 


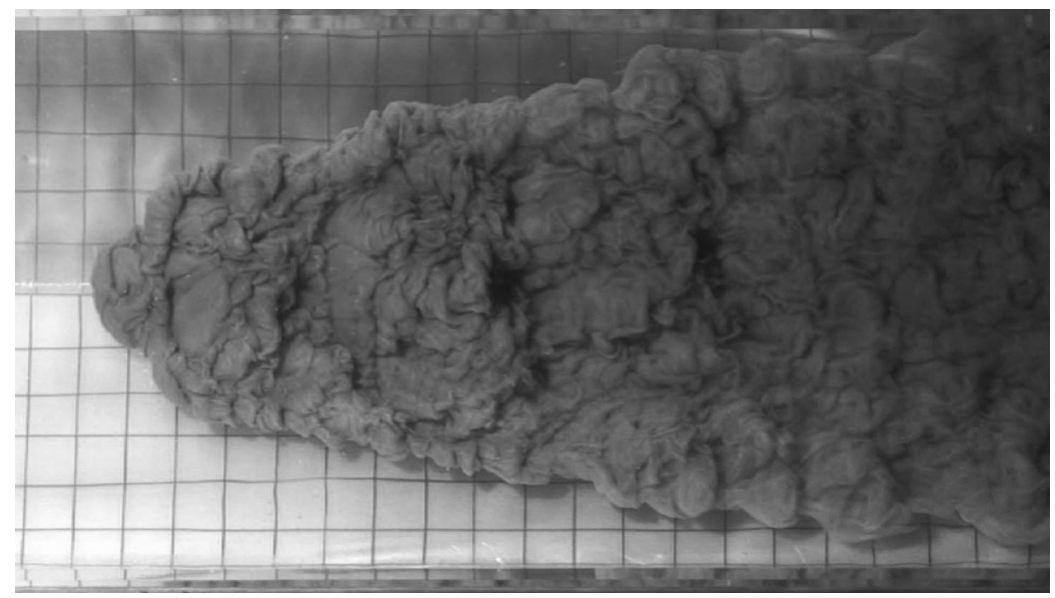

Figure 4. A close-up of the head of particulate gravity current, seen from above, flowing along a $\mathrm{V}$-shaped valley. The topography of the turbulence is similar to a rugged mountainous region separated by level plains about $2 \mathrm{~cm}$ wide.

of $h_{0}$. In contrast to the case of the flat-bottomed tank, the turbulence is maintained for a much larger distance away from the head. Figure 4 is a close-up of the front of a gravity current. Noticeable features of the turbulence are the flat-looking regions with a typical length scale of about $2-3 \mathrm{~cm}$ surrounded by large disordered regions with a typical length scale of $1 \mathrm{~cm}$.

Studies of the velocity field in saline gravity current experiments along flat-bottomed tanks show that strong fluctuations in the velocity field are associated with the billows generated by Kelvin-Helmholtz instabilities generated as the front rolls up (Kneller, Bennett \& McCaffrey 1997). The analog three-dimensional direct numerical simulations (DNS) of planar gravity current in the lock-exchange configuration and finite volume release (Cantero et al. 2008) show this in detail for Reynolds numbers in the range 8950 to 15000 using between $31 \times 10^{6}$ and $131 \times 10^{6}$ grid points. In particular the numerical calculations show that the downstream velocity is partly determined by the complex interaction between Kelvin-Helmholtz vortices. Saline gravity currents in a V-shaped valley differ from their counterpart in the flat-bottomed valley because Kelvin-Helmholtz billows propagate around the sides of the current as well as over the top. This difference in the dynamics of the interface between the gravity current and the ambient fluid may be the cause of the apparent difference in the turbulence in the flat and V-shaped-valley experiments. Finally, we note that the numerical simulations of Cantero et al. (2008) reports that the speed of the front is predicted equally well by two and three-dimensional simulations during the initial and slumping phases but begin to diverge in the inertial and viscous phases.

\section{Flat-bottomed tanks}

In order to provide a comparison with the gravity currents in a V-shaped valley two of the experiments used a flat-bottomed tank. In the following we describe the box model and the experimental results for this case.

\subsection{Box model for the flat-bottomed tanks}

Box-models (Huppert \& Simpson 1980; Dade \& Huppert 1995a) assume the gravity current remains homogeneous, the upper surface is horizontal, there is little mixing between the current and the ambient fluid, and the speed of the front can be obtained 
from a Froude number condition using the height of the current as a length scale. It is assumed that the main average velocity is along the tank and constant across the tank. In the case of particulate currents we make the further assumption (also used by Bonnecaze et al. 1993) that the current is sufficiently turbulent to ensure that the particulate mass density is nearly uniform, and that the deposit formula of Martin \& Nokes $(1998,1989)$, when suitably generalized, can be used.

Martin \& Nokes $(1988,1989)$ deduced their deposit equation from experiments and theory for turbulent thermal convection in a flat-bottomed container. They assumed that, in any time $\delta t$, the particulate matter $\delta m$ in a layer of thickness $v_{s} \delta t$ (where $v_{s}$ is the sedimentation velocity) adjacent to the bottom of the tank would be deposited. For a flat-bottomed tank, with height of current $h$, this represents an area fraction $v_{s} \delta t W /(h W)$, which is the negative of the mass fraction $\delta m / m$ and leads to the deposit equation

$$
\frac{\mathrm{d} m}{\mathrm{~d} t}=-\gamma \frac{m v_{s}}{h} .
$$

Martin \& Nokes $(1988,1989)$ found that the values of the factor $\gamma$ were in the range $0.6<\gamma<1.1$. The lower values occurred for values of the ratio of $v_{s} / v>0.5$, where $v$ is the root mean square velocity at the mid-point of their tank. Martin and Nokes pointed out that smaller values of $\gamma$ may be associated with the non-uniform mixing they observed in some experiments. Although it has become customary to use $\gamma$ equal to 1 we should expect that $\gamma$ will vary with the mixing process associated with gravity current and be space and time dependent.

In the case of saline gravity currents the mixing and entrainment in the head is known to differ from that along the tail of the current (Hacker et al. 1996; Hallworth et al. 1996) and to depend on the aspect ratio (height to length) of the lock (Hacker et al. 1996). Although these results are for saline currents, they suggest that the mixing in particulate currents may vary along the current, and may depend on the aspect ratio of the lock and in this way affect the deposit. This is confirmed for the case of particulate currents in flat-bottomed tanks for which the rate of mass deposit has a maximum under the head of the current (De Rooij \& Dalziel 2001, and unpublished experiments of De Rooij, Dalziel and Linden, private communication). We therefore expect that a box model, which assumes the rate of deposit is uniform along the current with a constant $\gamma$, would predict the final deposit and the speed of the head of the current with only moderate accuracy. This is the case for the box model results for flat-bottomed tanks shown below, and those of Bonnecaze et al. (1993), for which the predicted deposit is too high for the first few lock lengths. The box model does, however, give a good estimate of the deposit beyond this distance, and predicts the velocity of the head of the current with satisfactory accuracy.

When the gravity current flows in a tank with a V-shaped bottom the turbulence in the current, and hence the mixing appears to be nearly uniform along the current (see for example figure 4). This suggests that, in this case, a single value of $\gamma$ might be appropriate for the entire current. We will show that for the majority of the experiments in the V-shaped valley we get good agreement between the box models and the experiments using $\gamma=0.7$.

We denote the coordinate of the front measured from the end of the tank by $X$ and assume the velocity of the front is

$$
\frac{\mathrm{d} X}{\mathrm{~d} t}=F_{r} \sqrt{g^{\prime} h},
$$

where $F_{r} \sim 1$ is a Froude number, and $h$ is the height of the current. 
We use an estimate of $v_{s}$ valid for the particle Reynolds number $\mathscr{R} \lesssim 1$ and arbitrary $\varphi$. For the former we take an arithmetic average of the values for $\ln C_{D}$ given by Batchelor (1967) for the first- and second-order approximations for the drag $C_{D}$. For the latter we note from the results of Segrè et al. (2001) that the theoretical estimates of the fluctuations in the sedimentation velocity require a factor $(1-\varphi)^{5}$. The final result is

$$
v_{s}=\frac{d^{2} g\left(\rho_{p} / \rho_{a}-1\right)(1-\varphi)^{5}}{18 v(1+3 \mathscr{R} / 32)} .
$$

where $d$ is the diameter of a typical particle, $v$ is the kinematic viscosity coefficient of the ambient fluid and the particle Reynolds number $\mathscr{R}$ is defined by

$$
\mathscr{R}=\frac{d v_{s}}{v} .
$$

Because $v_{s}$ depends on $\varphi$ it changes during the flow. In our experiments, where $\varphi_{0} \lesssim 0.02$ this change is $\sim 10 \%$ and the effect of the factor $(1-\varphi)^{5}$ is small, but in other experiments with higher values of $\varphi$ it would be essential to include this factor.

In the case of the flat-bottomed tank the fixed volume $V$ of the current is given by

$$
V=h X W,
$$

and the longitudinal cross-section $A$ is $V / W$. Substituting this in (4.1) we obtain

$$
\frac{\mathrm{d} X}{\mathrm{~d} t}=F_{r} \sqrt{\frac{m \bar{g} A}{M X}},
$$

where $\bar{g}=g\left(1-\rho_{a} / \rho_{p}\right)$. If the variation of $v_{s}$ with $\varphi$ is neglected we can find the variation of $m$ with $X$ by taking the ratio of (4.1) to (4.2) and integrating to get

$$
m=k_{1}^{2}\left(X_{R F}^{5 / 2}-X^{5 / 2}\right)^{2},
$$

where

$$
k_{1}=\frac{\gamma v_{s}}{5 F_{r}}\left(\frac{M}{\bar{g} A^{3}}\right)^{1 / 2},
$$

while the run out distance $X_{R F}$ is given by

$$
X_{R F}=\left(L^{5 / 2}+\frac{m_{0}^{1 / 2}}{k_{1}}\right)^{2 / 5} .
$$

If we define a length scale for the flat-bottomed valley by $\ell_{F}=A^{1 / 2}$, and neglect the contribution from the term $L^{5 / 2}$, we can write $X_{R F}$ in the form

$$
X_{R F}=\ell_{F}\left(\frac{m_{0}}{M}\right)^{1 / 5}\left(\frac{5 F_{r}}{\gamma}\right)^{2 / 5}\left(\frac{\ell_{F} \bar{g}}{v_{s}^{2}}\right)^{1 / 5},
$$

where it is useful to note that $F_{r}$ and $\gamma$ occur in the combination $F_{r} / \gamma$, and the last factor involves a sedimentation Froude number. In the case of axisymmetric particulate currents, run out distances were estimated by Dade \& Huppert (1995b).

We solve (4.1) and (4.2) numerically using an integrator with errors $(\delta t)^{3}$ per time step. The deposit was calculated in the following way. The bottom of the tank was divided into strips of width $\Delta x$ and, at the end of each time step, the total mass deposited $\Delta m$ during the time step was assigned equally to each strip $k$ where $1 \leqslant k \leqslant X / \Delta x$. If $m_{k}$ is the final mass in strip $k$, the mass per unit area at the position of the strip is $m_{k} /(W \Delta x)$. 

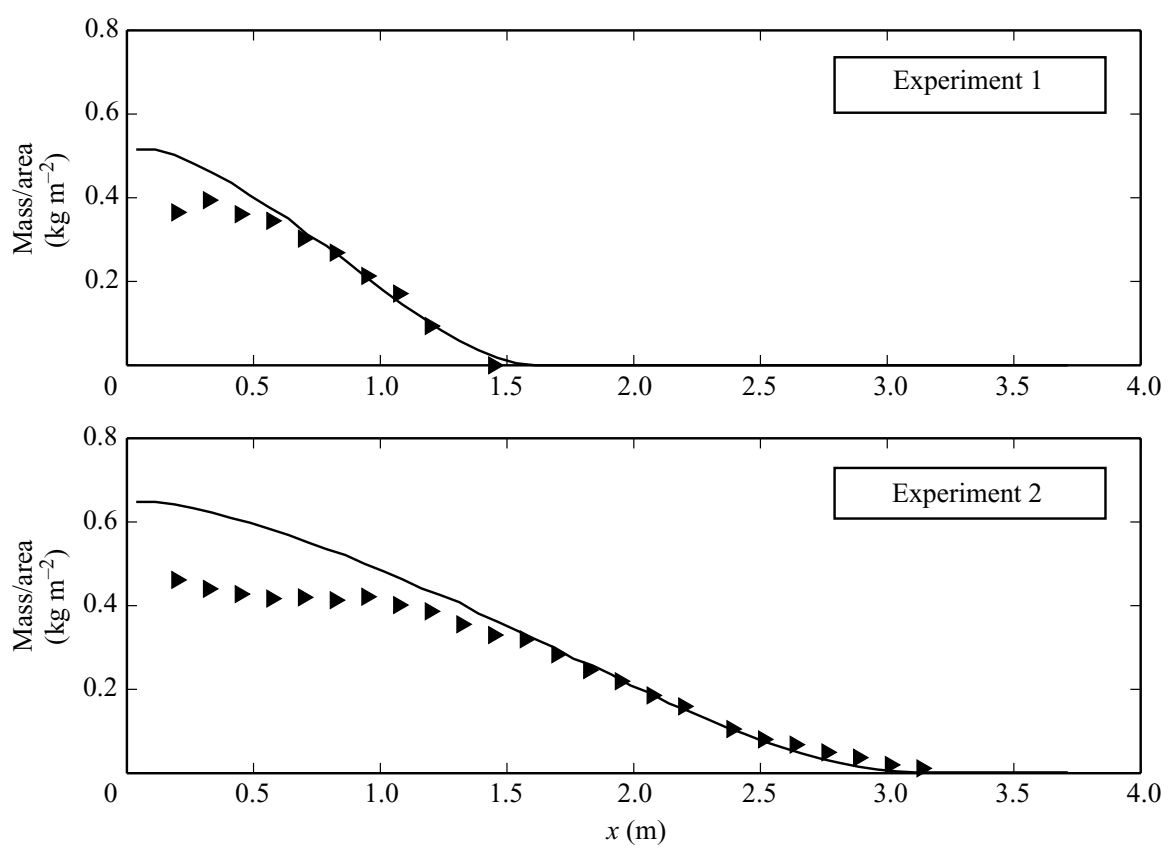

FIGURE 5. The mass/area against distance $x$ along the tank for our experiments 1 and 2 (flat-bottomed tank). The filled triangles are the experimental results and the results of the box model are shown by the continuous curves. The key parameters associated with these and all other experiments are in table 1. Here, and in all figures, SI units are used.

\subsection{Experiments in the flat-bottomed tank}

Figure 5 shows the variation of the mass deposited per unit area with distance along the flat-bottomed tank (experiments 1 and 2). The symbols show the experimental results and the continuous curve shows the box model results obtained using $\gamma$ equal to 0.7 and 0.5 for experiments 1 and 2, respectively. A characteristic feature of the experimental mass deposit is the nearly flat profile for a distance of a few lock lengths, the indication of a peak near $x=0.4$ for experiment 1 and near $x=1$ for experiment 2 , followed by a monotonic decrease for both experiments. The deposit predicted by the box model is significantly higher than the experimental data over the first few lock lengths.

The run out lengths $X_{R F}$ can be calculated using the following parameters for experiment 1 . These are $\ell_{F}=0.0798 \mathrm{~m}$ and, if the initial value of $\varphi$ is used in the definition of $v_{s}$, then $\bar{g} \ell_{F} / v_{s}^{2}=1.72 \times 10^{6}$, giving $X_{R F}=1.55 \mathrm{~m}$. If the factor $(1-\varphi)^{5}$ is replaced by 1.0 then $\bar{g} \ell_{F} / v_{s}^{2}=1.83 \times 10^{6}$ and $X_{R F}=1.62 \mathrm{~m}$.

For experiment 2 we find $\ell_{F}=0.122 \mathrm{~m}$ and, if the initial value of $\varphi$ is used in the definition of $v_{s}$ then $\bar{g} \ell_{F} / v_{s}^{2}=2.278 \times 10^{6}$, giving $X_{R F}=2.70 \mathrm{~m}$. If the factor $(1-\varphi)^{5}$ is replaced by 1.0 then $\bar{g} \ell_{F} / v_{s}^{2}=2.25 \times 10^{6}$, giving $X_{R F}=2.59 \mathrm{~m}$. The differences in $X_{R F}$ produced by including or excluding the initial values of $\varphi$ in the calculation of $v_{s}$ are small, and typically $4 \%$. Reference to figure 5 shows that these box model estimates are satisfactory.

The variation of $X$ with distance $x$ along the tank is shown in figure 6 for experiments 1 and 2 . It can be seen that the results for experiment 2 are in excellent agreement with the box model whereas those for experiment 1 are less satisfactory. We note that the results of Bonnecaze et al. (1993) for particles with diameters in the 

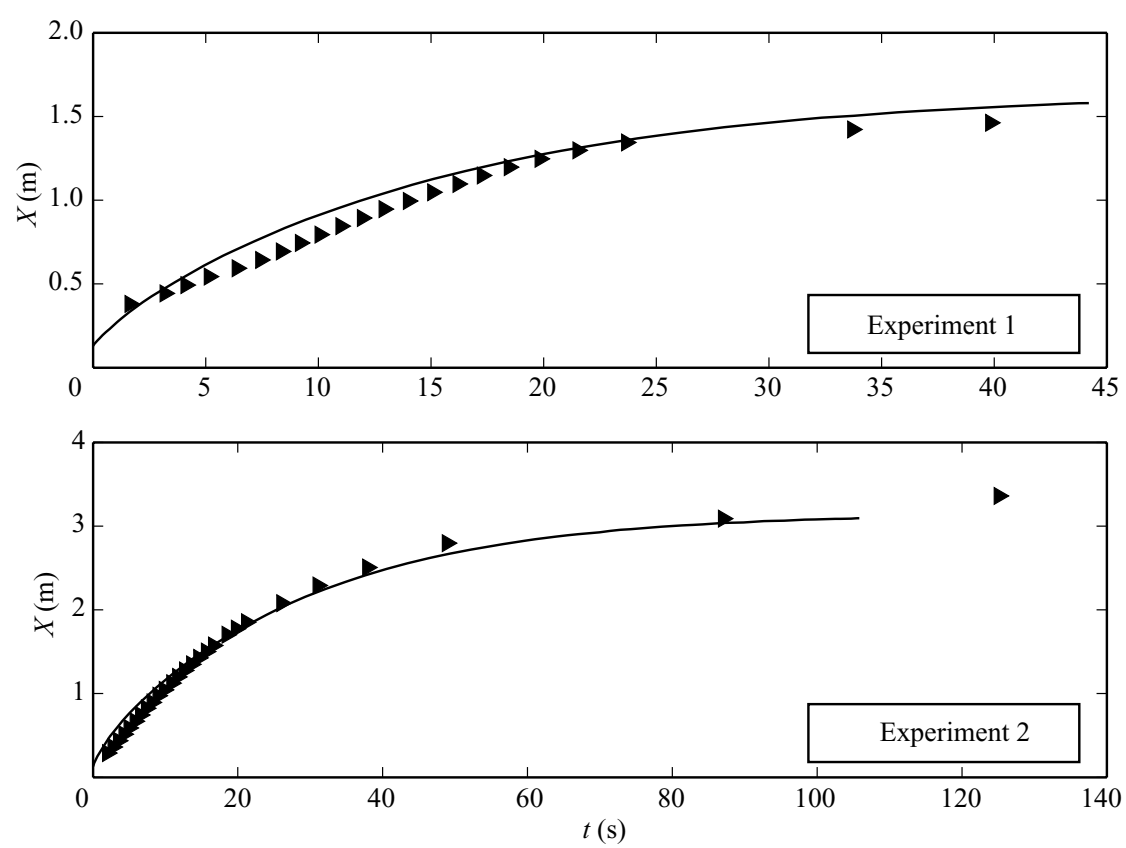

Figure 6. The position of the head of the current $X$ against time for a particulate gravity current flowing over a flat-bottomed valley for experiments 1 and 2 . The filled triangles are the results from our experiments. The continuous curves are the present box model.

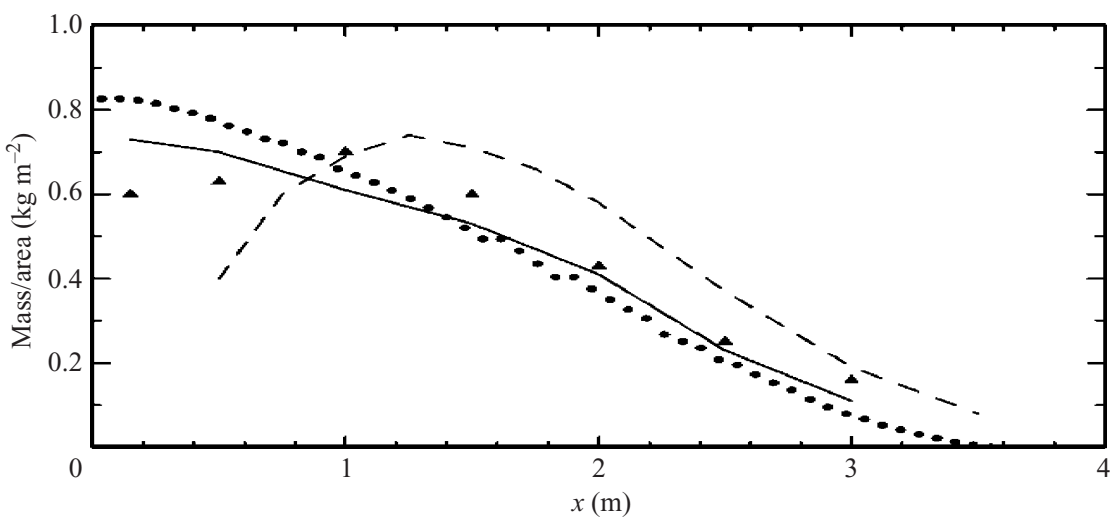

FIGURE 7. The mass/area against distance $x$ along the flat-bottomed tank for a particulate gravity current carrying particles of diameter $53 \mu \mathrm{m}$. The filled triangles are the experimental results of Bonnecaze et al. (1993). The black dots are the present box model. The continuous curve shows the results of a numerical solution by Bonnecaze et al. and the dashed curve shows the results of an approximate box model due to Dade \& Huppert (1995a).

range 23-37 microns show similar discrepancies between model and experiment for $X$ as a function of distance $x$ along the tank (see their figures 11 and 12).

Figure 7 shows a direct comparison between some experimental data and models of Bonnecaze et al. (1993) with the above box model within general agreement. This figure shows that the deposit profile has the characteristic plateau for distances of several lock lengths after which it falls monotonically to zero as in our experiments. The figure also shows the theoretical results obtained by Bonnecaze et al. (1993) using 
a two layer model, and the results of Dade \& Huppert (1995a) using an approximate box model. For this case our box model gives a good fit with $\gamma=0.3$. We do not know why the best fit $\gamma$ is smaller for this case than in our experiments.

\section{V-shaped valleys}

In this section we generalize the box model to apply to the V-shaped valley. Following the discussion in $\S 4$ we expect that the mixing in the case of V-shaped valleys will be more uniform than for the flat-bottomed valleys and that the box model assumptions will be more accurate. This is verified by comparison with experiment as shown below.

\subsection{Box model for the $V$-shaped valley}

The box model for a V-shaped valley has the same set of equations as before, except that the height of the current $h$ is now measured from the bottom of the valley. Again, the velocity is assumed to be the same across the valley, and the deposit at any time to be the same across that part of the valley reached by the current. Hence the assumption of a two-dimensional flow. The change in depth across the valley is taken care of through the relations between the geometry, the depth of the current and the volume of the current. When seen from above the box model approximates the current at any time by a rectangle which becomes longer and narrower as the flow proceeds. These assumptions have been shown to be satisfactory in the case of saline currents (Monaghan et al. 2008).

Provided the initial water level is not below the edge of the valley, the volume $V=W L\left(h_{0}-a / 2\right)$ and the longitudinal cross-section $A=L\left(h_{0}-a / 2\right)$. When $h \geqslant a$

$$
h=\frac{a}{2}+\frac{V}{W X}=\frac{a}{2}+\frac{A}{X},
$$

and when $h<a$

$$
h=\sqrt{\frac{2 a V}{X W}}=\sqrt{\frac{2 a A}{X}} .
$$

The gravity currents in our experiments are turbulent but, as noted by Martin \& Nokes $(1998,1989)$, the turbulence becomes negligible close to the boundary because both the normal and tangential velocities are zero on the boundary. We do not know if the turbulence has the same energy and spectrum across the valley, nor whether the sedimentation velocity entering the non-turbulent boundary is necessarily vertical, nor whether the non-turbulent boundary has the same thickness across the valley. The simplest assumption is that the direction of $v_{s}$ is vertical and is identical across the valley. The component of $v_{s}$ perpendicular to the walls of the valley is then $v_{s} \cos \theta$ where $\tan \theta=2 a / W$, and $\theta$ is the angle of the valley side to the horizontal. Provided $h \geqslant a$, each side of the valley has length $s=W /(2 \cos \theta)$ and the cross-sectional area from which mass deposit occurs is $2 s v_{s} \cos \theta \delta t$. The total cross-sectional area of the valley is $W(h-a / 2)$. The fraction of the mass in the current which is deposited on the valley in time $\delta t$ is then given by

$$
\frac{|\delta m|}{m}=\frac{\text { Area of deposit }}{\text { Total area }}=\frac{\left(2 s v_{s} \cos \theta \delta t\right)}{W(h-a / 2)},
$$

or

$$
\frac{|\delta m|}{m}=\frac{v_{s} \delta t}{(h-a / 2)} .
$$


Taking account of the sign, and including the factor $\gamma$ as in (4.1), we obtain the equation for the mass deposit when $h \geqslant a$

$$
\frac{\mathrm{d} m}{\mathrm{~d} t}=-\frac{\gamma m v_{s}}{(h-a / 2)}
$$

If $h \leqslant a$ we find, following the previous argument, that

$$
\frac{\mathrm{d} m}{\mathrm{~d} t}=-\frac{2 \gamma m v_{s}}{h} .
$$

As before we can determine the variation of $m$ with $X$ by assuming there is no $\varphi$ variation in $v_{s}$ then taking the ratio of $\mathrm{d} m / \mathrm{d} t$ and $\mathrm{d} X / \mathrm{d} t$ and integrating. When $h>a$, we find that

and

$$
\frac{\mathrm{d} m}{\mathrm{~d} X}=-\frac{\gamma v_{s} \sqrt{M}}{F_{r} \sqrt{\bar{g}}} \frac{m^{1 / 2}}{(h-a / 2) h^{1 / 2}},
$$

$$
h=\frac{a}{2}+\frac{A}{X} .
$$

Substituting for $h$ in terms of $X$ from (5.8), and solving the resulting differential equation, we obtain

$$
m^{1 / 2}=k_{2}\left\{q^{3 / 2}(1+q)^{1 / 2}-\frac{3}{8}\left(q^{1 / 2}(1+q)^{1 / 2}(1+2 q)-\ln \left(q^{1 / 2}+\sqrt{1+q}\right)\right)\right\}+c
$$

where $c$ is an arbitrary constant, $q=X / X_{v}, X_{v}$ is the value of $X$ when $h=a$ and $k_{2}$ is given by

$$
k_{2}=\frac{\gamma v_{s}}{F_{r}}\left(\frac{2^{5} M A^{2}}{\bar{g} a^{5}}\right)^{1 / 2} .
$$

The arbitrary constant $c$ is determined by the condition that, when $x=L, m$ is the initial mass of particles in the current.

When $h<a$ we find that

$$
m=k_{3}^{2}\left(X_{R V}^{7 / 4}-X^{7 / 4}\right)^{2},
$$

where $X_{R V}$ is the run out distance and $k_{3}$ is given by

$$
k_{3}=\frac{4 v_{s} \gamma}{7 F_{r}}\left(\frac{M^{2 / 3}}{2 a A \bar{g}^{2 / 3}}\right)^{3 / 4} .
$$

Accordingly, if the flow is entirely in the valley then

$$
X_{R V}=\left(L^{7 / 4}+\frac{m_{0}^{1 / 2}}{k_{3}}\right)^{4 / 7} .
$$

If the term involving $L$ is neglected, and a length scale defined by $\ell_{V}=(2 a A)^{1 / 3}$, then we can write $X_{R V}$ in the form

$$
X_{R V}=\ell_{V}\left(\frac{7 F_{r}}{4 \gamma}\right)^{4 / 7}\left(\frac{m_{0}}{M}\right)^{2 / 7}\left(\frac{\bar{g} \ell_{V}}{v_{s}^{2}}\right)^{2 / 7},
$$

where, as in the case for the flat-bottomed valley, the last factor is a Froude number involving $v_{s}$ though this time with a different length scale. As in the case of flatbottomed tanks the parameters $F_{r}$ and $\gamma$ occur in the combination $F_{r} / \gamma$. 
Using (4.10) and (5.14), we can compare the run out length between currents in a flat-bottomed tank and currents in a V-shaped valleys for similar initial conditions. An example relevant to the present experiments is to assume the current in the $\mathrm{V}$-shaped valley is always in the valley and extends to upper edge of the valley (as for experiment 3). If we assume that, in both cases, the initial volume of the current $V$, the initial width $W$ and the length the lock $L$ are the same, $V=1 / 2 a W L$ with

$$
\ell_{v}=(2 a A)^{1 / 3}=\left(\frac{4 V^{2}}{W^{2} L}\right)^{1 / 3}
$$

and

$$
\ell_{F}=(V / W)^{1 / 2}
$$

The ratio of run out length for the $\mathrm{V}$-shaped valley $X_{R V}$ to that for the flat-bottomed tank $X_{R F}$ is then given by

$$
\frac{X_{R V}}{X_{R F}}=\left(\frac{7^{20}}{5^{14} 2^{10}}\right)^{1 / 35}\left(\frac{\bar{g} m_{0}}{M v_{s}^{2}}\right)^{3 / 35}\left(\frac{F_{r}}{\gamma}\right)^{6 / 35}\left(\frac{1}{L^{15}}\left(\frac{V}{W}\right)^{9}\right)^{1 / 35}
$$

It can be seen that, for the above conditions, the ratio of runout distances for the $\mathrm{V}$-shaped and flat-bottomed valleys increases as $V / W$ increases, but decreases as the lock length $L$ increases. The ratio also increases as the initial effective gravity $\bar{g} m_{0} / M$ increases, and decreases as the sedimentation velocity $v_{s}$ increases as it would do for coarser particulate matter. The initial velocity of the current is larger in the case of the $\mathrm{V}$-shaped valley because, for the same $V$ and maximum width $W$, the height of the fluid $h$ is less for the flat-bottomed tank. For the above conditions, the initial height of the fluid in the flat-bottomed tank is half the initial height of the fluid in the $\mathrm{V}$-shaped valley.

In practice we find it more convenient to determine $m$ as a function of $x$ by integrating the original differential equations giving $m$ and $X$ as functions of $t$ rather than use the previous algebraic expressions, though they provide convenient checks of the numerical algorithm. As before, the equations were integrated using a mid-point predictor-corrector algorithm with errors per time step $O\left(\delta t^{3}\right)$. The results to be described use a Froude number $F_{r}$ of 1.0 and the values of $\gamma$ given in table 1 (see the Appendix for further discussion of the sensitivity of the results to the values of $\gamma$ and $F_{r}$ ). The deposit was calculated by first dividing the valley into strips (perpendicular to the line of the bottom of the valley) and the strips into slices. At any time the mass deposited was divided equally amongst the slices which the current could reach. Initially the current occupies the entire width of the valley and can deposit onto all the slices of those strips reached by the head of the current. Eventually the current is confined entirely to the valley and the more extended the current the narrower it must be. The deposit then extends a decreasing distance up the sides of the valleys. The result is that the deposit is concentrated in the central regions of the valley.

\subsection{Experiments in the $V$-shaped valley}

Figure 8 shows the variation of the position of the head of the current against time for experiments 3 and 4. The box model results are shown by a continuous curve, and the experimental results are shown by filled triangles. The agreement is good. Figure 9 shows the experimental and theoretical results for the mass deposited per unit area as a function of distance $x$ along the tank. The agreement between theory and experiment is much better in this case than for the flat-bottomed tank. Note the difference in profile between that in figure 9 and those in figure 5 (flat bottom). In 

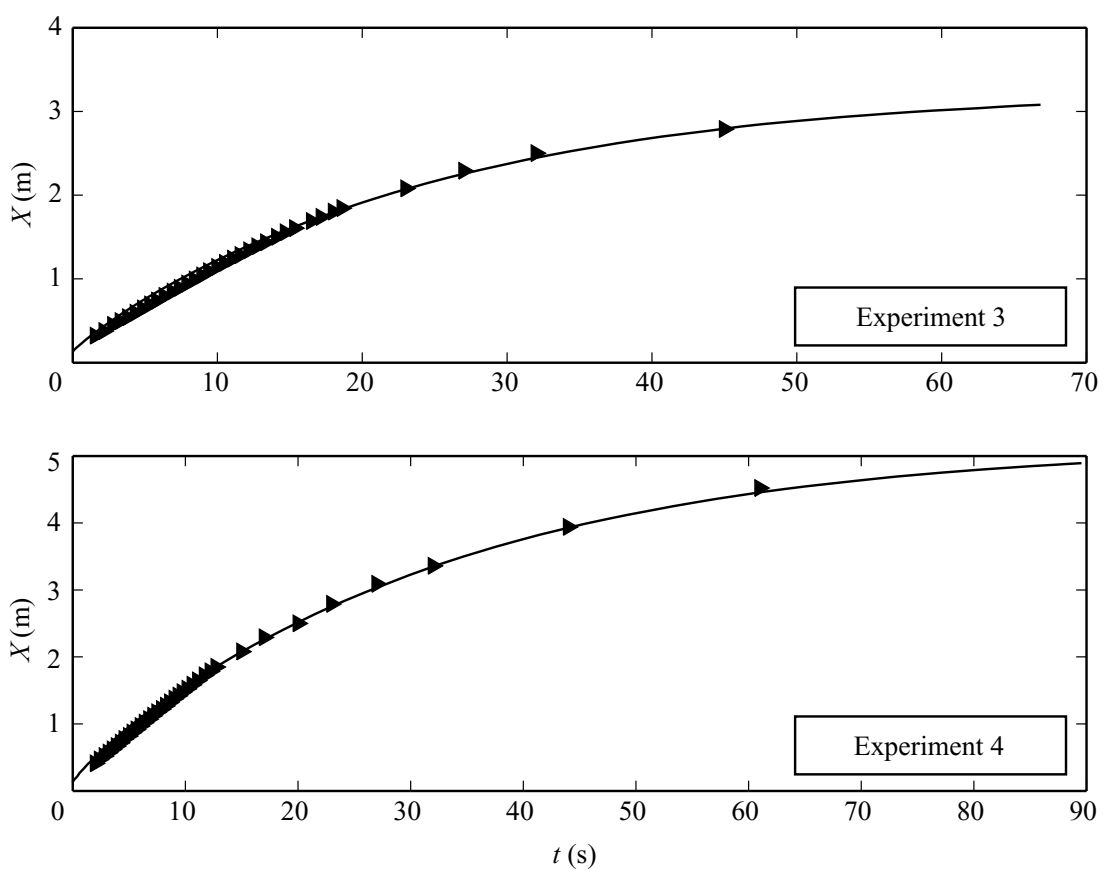

FIGURE 8. The position of the head of the current flowing along the V-shaped valley against time for our experiments 3 and 4 . The experimental results are shown by filled triangles and the box model results by a continuous curve.
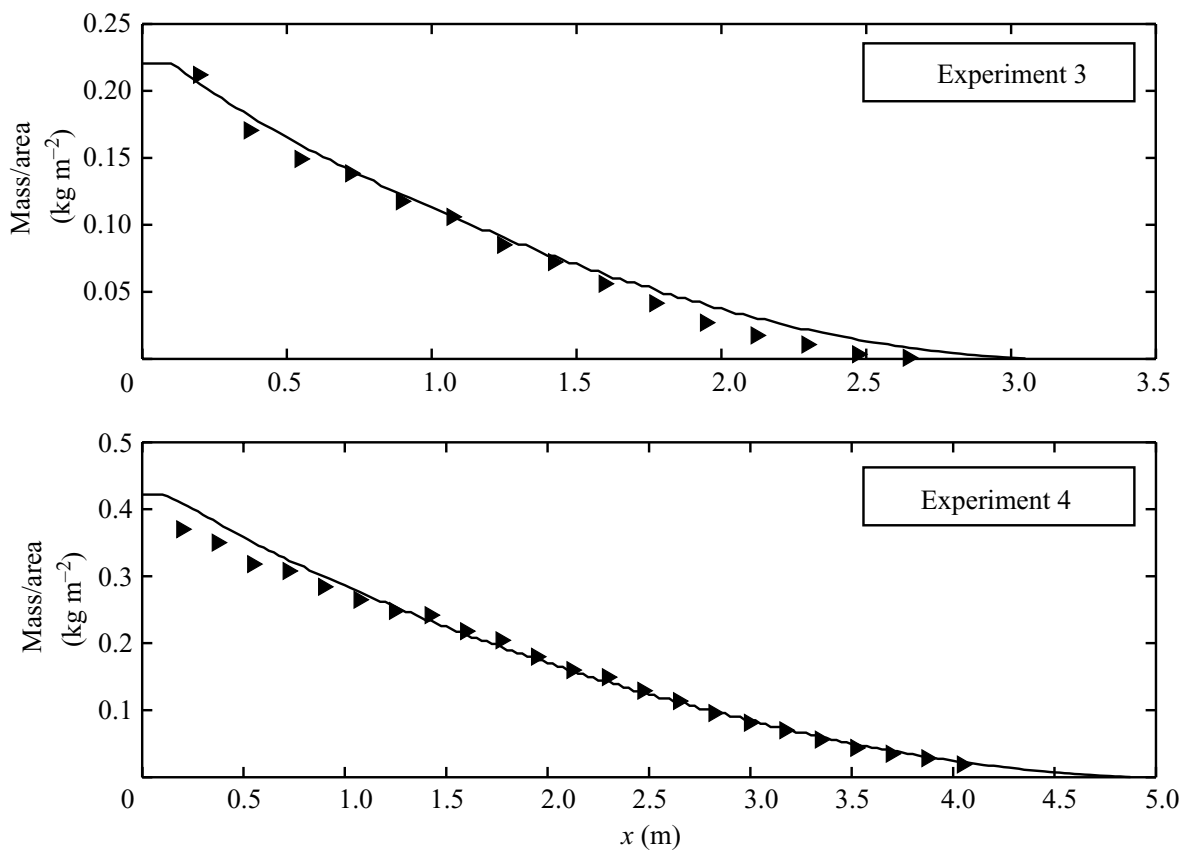

FIGURE 9. The mass deposit/area against distance $x$ for flow along the V-shaped valley for experiments 3 and 4 . The experimental results are shown by filled triangles and the box model by the continuous curves. The agreement between theory and experiment is much better for the V-shaped valley than for the flat-bottomed tank. 

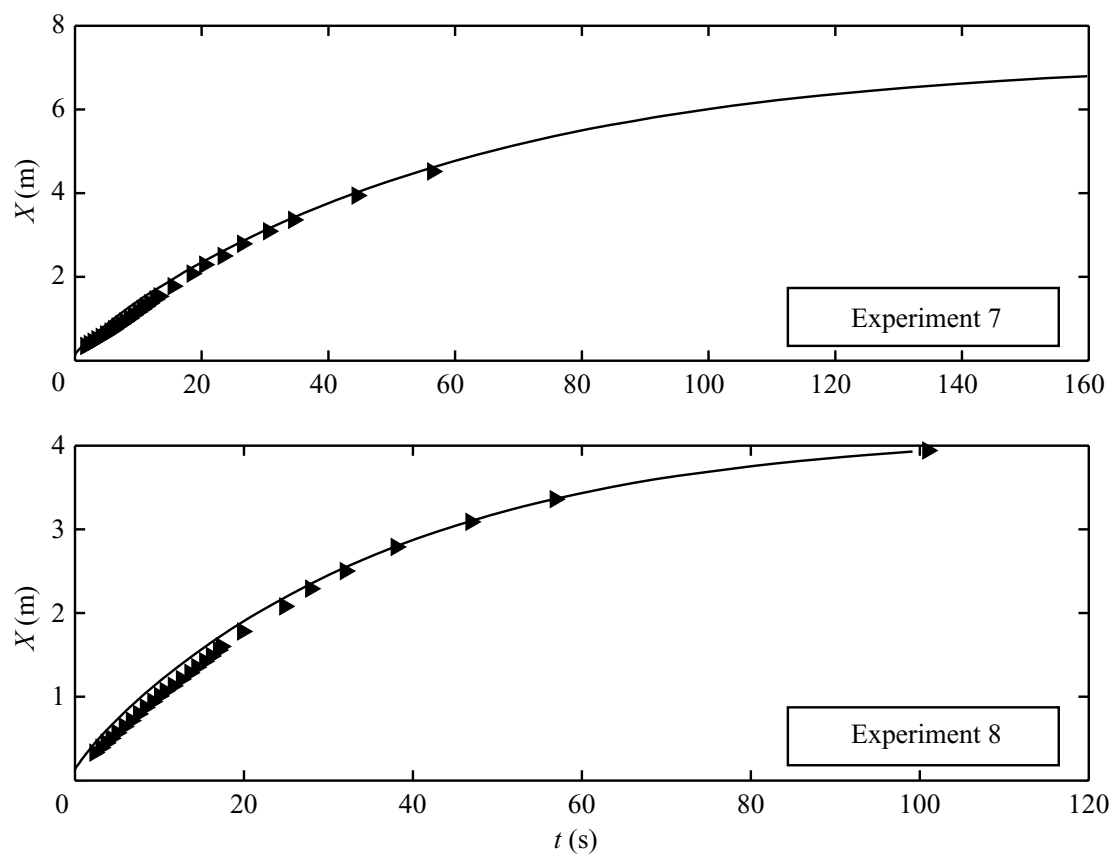

FIGURE 10. The position of the head of the current flowing along the V-shaped valley against time for our experiments 7 and 8. The experimental results are shown by filled triangles and the box model results by a continuous curve.

particular the absence in the figure of the flattened region for small $x$ and the slight peak evident in the results for the flat-bottomed tank in figures 5 .

The run out distance for experiment 3 calculated using the parameters $\ell_{V}=0.0827 \mathrm{~m}$, with the initial value of $\varphi$ is included in the definition of $v_{s}$ is $X_{R V}=3.37 \mathrm{~m}$. If the factor $(1-\varphi)^{5}$ in $v_{s}$ is replaced by 1.0 we find $X_{R V}=3.18 \mathrm{~m}$. This result is in good agreement with the results for experiment 3 shown in figure 9. The ratio $X_{R V} / X R F$ estimated using (5.15) is 2.60 , which shows that the run out distance is greatly extended by the $\mathrm{V}$-shaped valley compared to that for a current of the same initial volume flowing along a flat bottom. The formula for $X_{R V}$ cannot be applied to experiment 4 because in that experiment $h_{0}>a$.

The results for experiments 5 and 6 are similar to those shown for experiments 3 and 4. These experiments (3-6) all have $g_{0}^{\prime} \sim 0.45$ and $h_{0}$ values which result in the current being entirely within the V-shaped valley either at the start or after the current length is approximately two lock lengths.

Figure 10 shows time variation of the head of the current for both experiments and box models for experiments 7 and 8 . These experiments have lower $g_{0}^{\prime}$ than experiments 3-6, namely 0.23 compared to 0.45 , and the lock aspect ratios differ by a factor of 2. The results for the box model use $\gamma=0.5$ for experiment 7 and $\gamma=0.7$ for experiment 8 . The agreement between the experiments and the box model is very good.

Figure 11 shows the mass deposit profile for the two experiments. The agreement with experiment is good though the results for experiment 7 are significantly higher than those for the experiment within the first few lock lengths. Experiment 7 has the higher lock aspect ratio and the results suggest that the lock aspect ratio affects particulate gravity currents as it does for saline gravity currents. In this case the V-shaped valley is initially only a small perturbation to the flow. Over the first few 

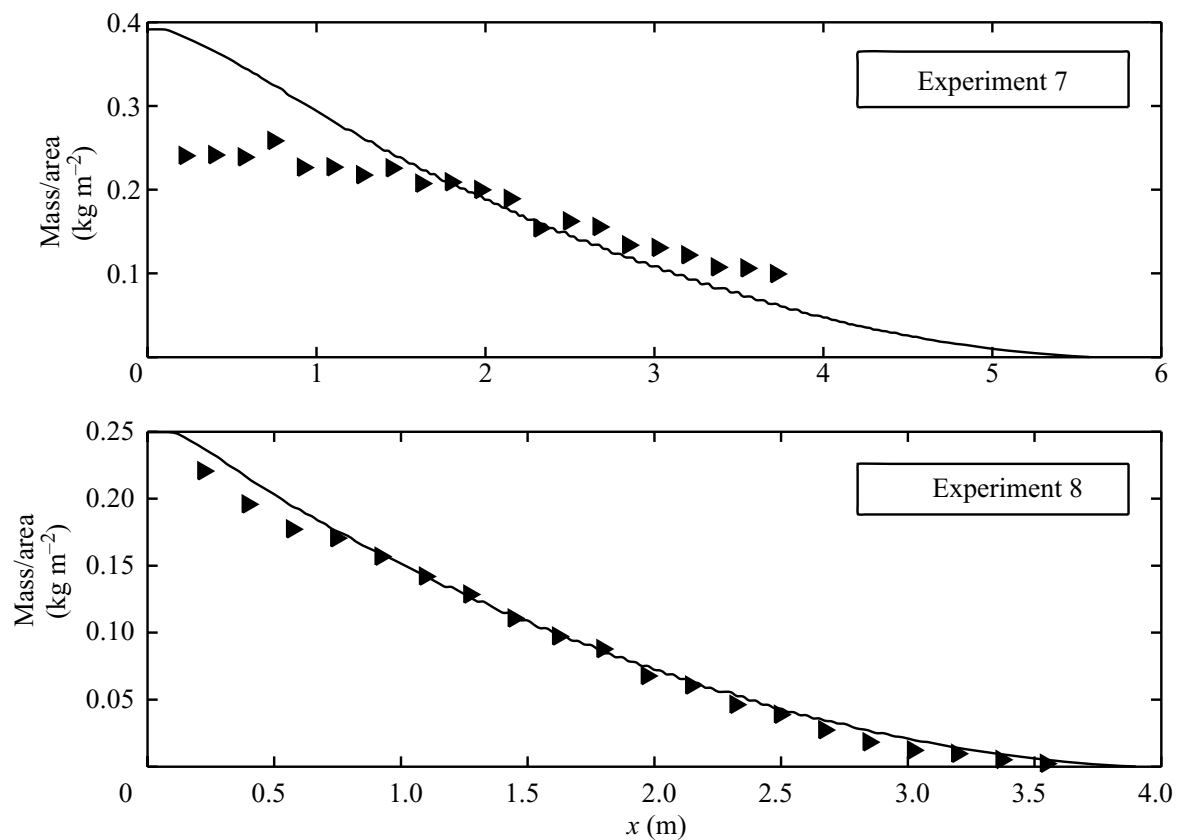

FiguRE 11. The mass deposit/area against distance $x$ for flow along the V-shaped valley for experiments 7 and 8 . The experimental results are shown by filled triangles and the box model by the continuous curves. Note that the lock aspect ratio of experiment 7 is twice that of experiment 8 .

lock lengths the flow is therefore similar to the flat-bottomed gravity current with its generic flattened deposit profile. Experiments 9 and 10 involve an effective gravity which is less than half that in the other experiments and experiment 9 has the same lock aspect ratio as experiment 7 . We noticed from the movies of the experiment that, immediately after the lock gate was lifted, the particulate current produced slowly moving eddies from the surface to the bottom of the valley. This continued for a few lock lengths after which the normal gravity current developed. This behaviour was most conspicuous for experiment 9 which has the largest aspect ratio. After this initial period a gravity current similar to those in the previous experiments developed.

Figure 12 shows the run of $X$ against $t$ for these two experiments. The agreement between the box model results and those from the experiments is reasonable but not as good as those from experiments 3-8. Figure 13 shows the mass deposit/area for experiments 9-10. The agreement between experiment and box model for experiment 10 is excellent. The agreement for experiment 9 is less satisfactory and this may be due to its higher lock aspect ratio.

The difference in the deposit across the valley is shown by figures 14 and 15 . There is a factor 4-5 difference in the magnitude of the mass per unit area in the centre region and on the flanks of the valley. This variation is quite different to that in a flat-bottomed tank whose deposits are uniform across the bottom. To determine if the greater deposit in the central region was due to particles sliding or rolling down the slope under gravity we performed simple experiments where a small amount of the $\mathrm{SiC}$ particles used in the experiments were released without and under water to sediment in the V-shaped valley. Once they were on the slope they showed no sign of moving down to the bottom of the valley even when subject to a disturbance. The simplest explanation of the deposit pattern is that, as the current extends down 

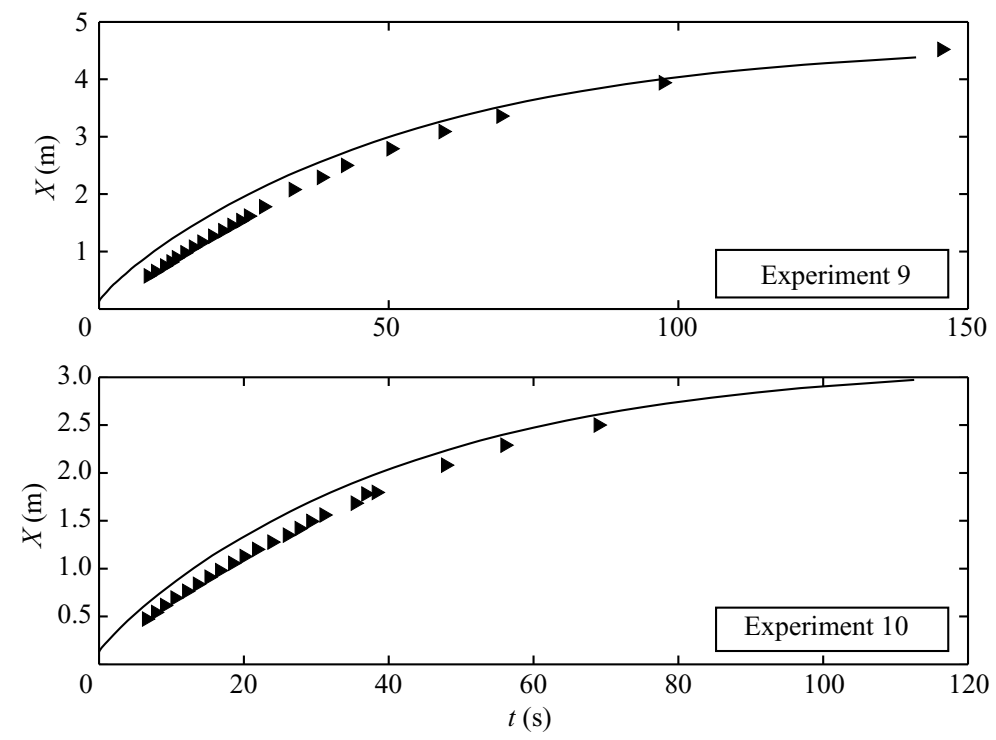

FIGURE 12. The position of the head of the current flowing along the $\mathrm{V}$-shaped valley against time for our experiments 9 and 10. The experimental results are shown by filled triangles and the box model results by a continuous curve.
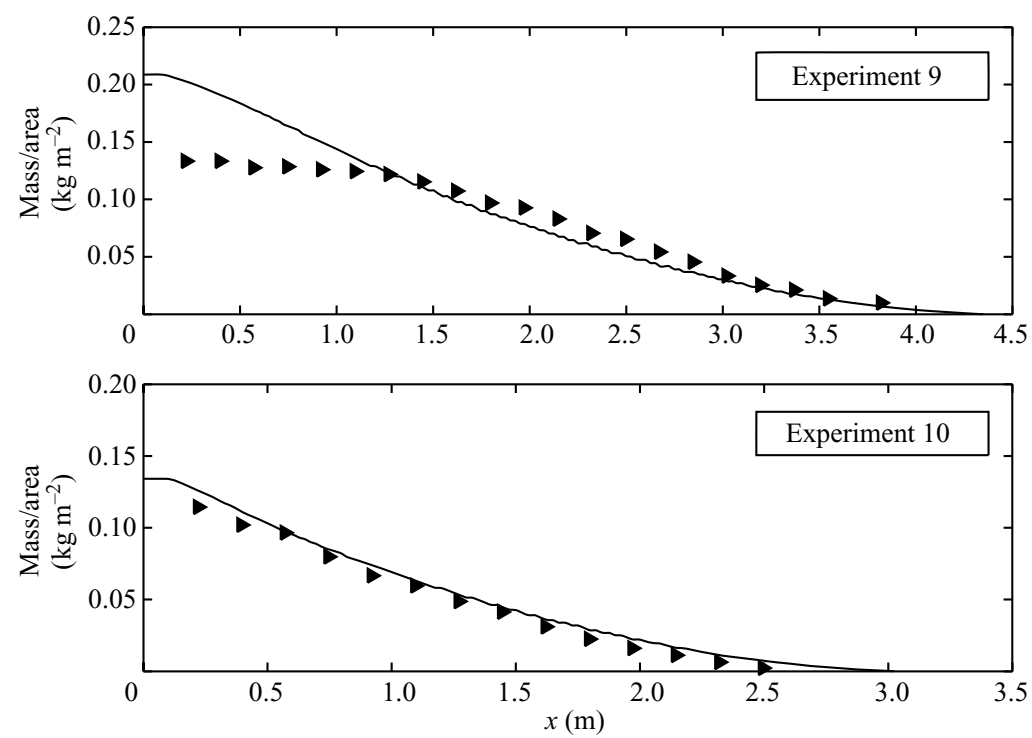

FIGURE 13. The mass deposit/area against distance $x$ for flow along the V-shaped valley for experiments 9 and 10. The experimental results are shown by filled triangles and the box model by the continuous curves. The lock aspect ratio of experiment 9 is twice that of experiment 10 .

the valley, it narrows, and is eventually unable to deposit outside the central region. This assumption is implicit in our box model calculations. Furthermore, despite the fact that the box model can only be a very crude model of the complex processes occurring in these experiments, it provides an excellent description of the variation in the deposit across the valley. 


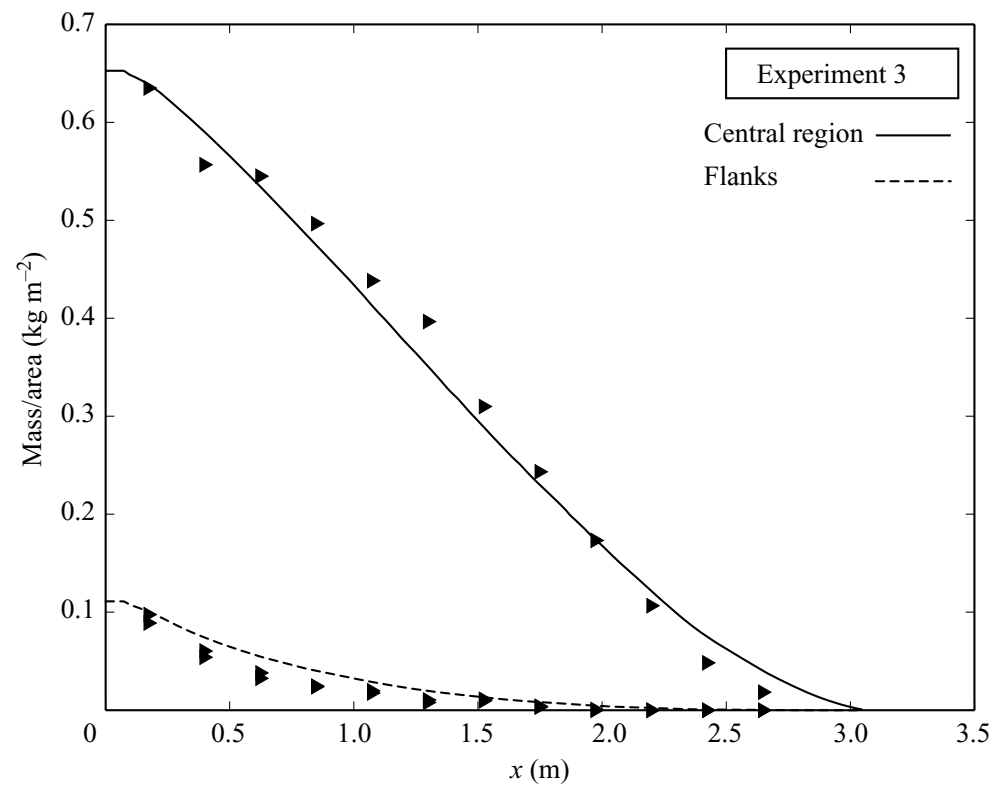

FIGURE 14. The mass deposit/area against distance for flow along the V-shaped valley for experiment 3. Strips were cut into three slices consisting of two symmetric flank sub-strips, each of length $12.6 \mathrm{~cm}$, and a central sub-strip, of length $6 \mathrm{~cm}$ (see figure 1). The experimental results are shown by filled triangles and the continuous and dashed curves are the mass/area predicted by the box model. The upper curve shows the results for the central region and the lower shows the results for the flanks. The experimental results for either flank are nearly identical.

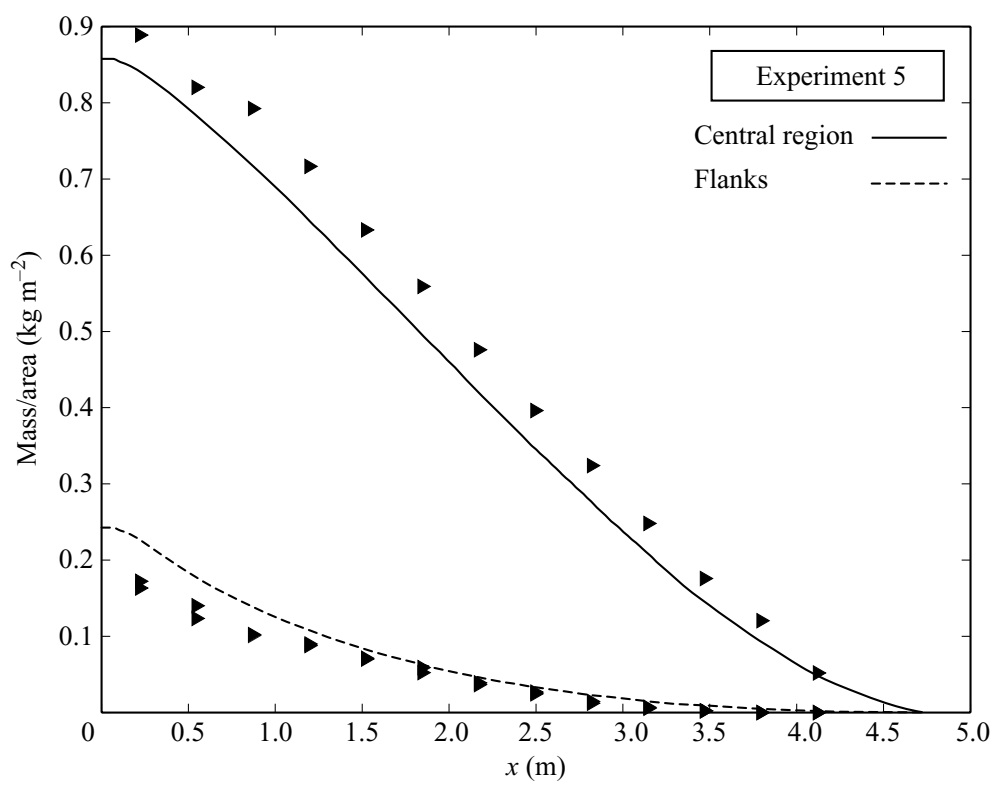

FIGURE 15. The mass deposit/area against distance for flow along the V-shaped valley for experiment 5 . The symbols have the same meaning as in figure 14 . 


\section{Discussion and conclusions}

The results of this paper show that the presence of the V-shaped valley causes significant changes to the deposit pattern compared with a flat-bottomed tank for constant volume particulate gravity currents. In the $\mathrm{V}$-shaped-valley experiments with lock aspect ratios $\sim 1$, the deposit monotonically decreases whereas, for the flat-bottomed tank, the deposit for $x<1 \mathrm{~m}$ is either flattened or shows a peak. The V-shaped-valley experiments 7 and 9, which have a high lock aspect ratio $\sim 2$, and initial height of the current a factor $\sim 4$ times the depth of the valley have mass deposit profiles similar to that for the flat-bottomed tanks. We believe that this is due to the $\mathrm{V}$-shaped valley having less influence on the deposit over the first few lock lengths of the flow when the height of the current is significantly greater than the depth of the valley.

Our experiments show that the mass/area deposit in the central part of the Vshaped valley (taken as that area within a strip of $7.6 \mathrm{~cm}$ length centred on the bottom of the valley) is considerably greater than that on either of the flanks of the valley. This appears to be largely due to the fact that, as the current extends down the valley, it narrows and is eventually unable to deposit outside the central region.

We have generalized the deposit equation of Martin and Nokes and used it with a box model to predict the speed of the front, the mass deposited along the valley and the difference between the deposits in the central regions and the flanks of the valley. For all these cases the box model gives predictions in very good agreement with experiments. The agreement is better in the case of the V-shaped valley than for the flat-bottomed tank. Our results show that the factor used in the deposition rate equation which is commonly assumed to be 1 should be smaller, typically 0.7 .

In addition to the changes in the deposit pattern produced by the V-shaped valley there are other changes to the flow. The first of these changes is the shape of the front when seen from above. When it is straight for the flat-bottomed valley, it is parabolic for the $\mathrm{V}$-shaped valley. This feature had also been found for saline gravity currents in a V-shaped valley (Monaghan et al. 2008). Associated with this is the obvious, but important, fact that the current becomes narrower as it extends further down the V-shaped valley. By contrast, in a flat-bottomed tank, the current always extends from one side of the tank to the other. The run out distance of the current in the case of the V-shaped valley also differs from that in the flat-bottomed valley. In the simple case of two particulate currents of equal volume, and equal initial upper surface width and for a V-shaped-valley current entirely in the V-shaped valley, we have derived the ratio of the run out distance in a V-shaped valley by the one in a flat-bottomed valley. Applied to our experiment 3, we predict that the $\mathrm{V}$-shaped valley run out distance is a factor 2.60 greater than that in the flat-bottomed valley.

There are numerous questions which remain to be answered. For example, it would be interesting to study gravity currents where the faceted $\mathrm{SiC}$ particles are replaced by nearly spherical glass beads which we expect to move down the sides of the valley more freely than $\mathrm{SiC}$ particles. A related question is how the dynamics of a gravity current, and the deposit from it, changes when the current contains particles of different sizes with very different sedimentation velocities. An important generalization is to the case where there are a small number of much larger particles since this configuration mimics pyroclastic or turbidity currents with accessory lithics (larger rocks and boulders). These processes are currently being studied in our laboratory.

This research was funded by an ARC Discovery grant of Monaghan and Huppert. The research of H. E. Huppert is partially supported by a Royal Society Research 

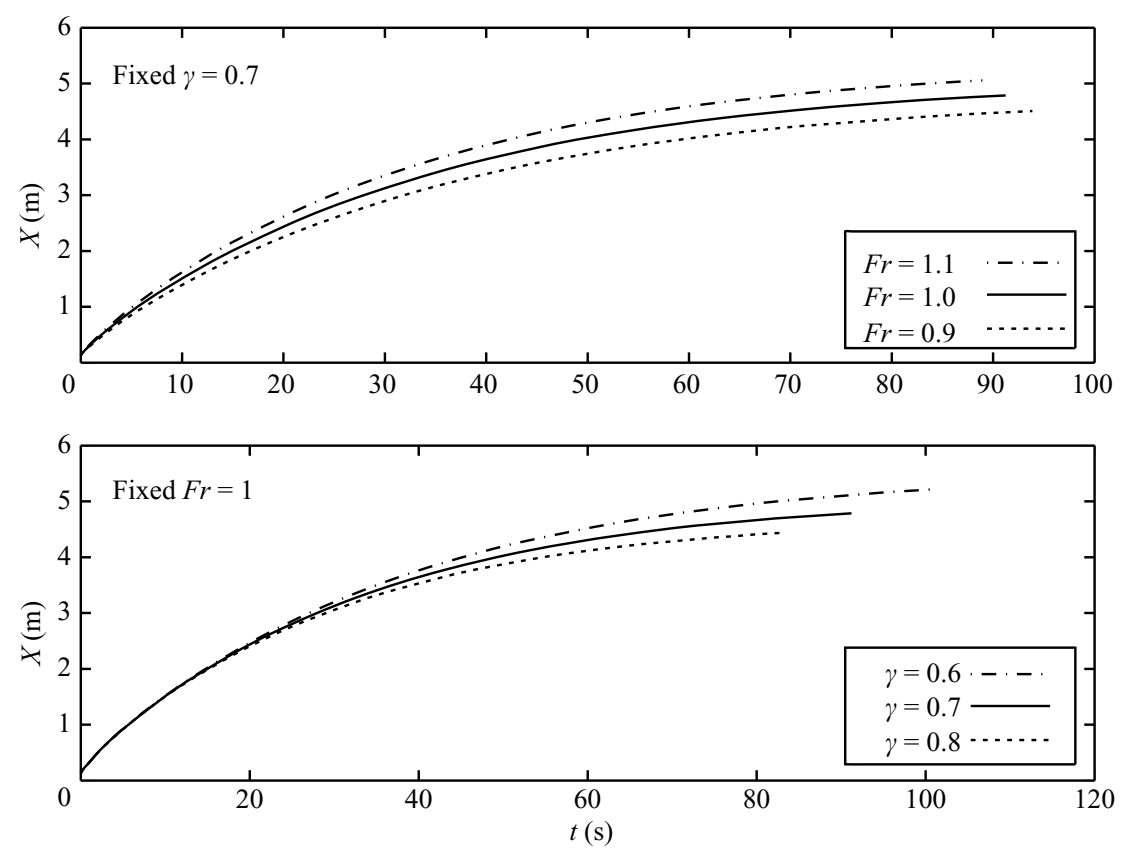

FIGURE 16. The $X, t$ curves for the values of $F_{r}$ and $\gamma$ shown.

Merit Award. Catherine Mérieux acknowledges financial support from ARC grant DP0449979.

\section{Appendix. Sensitivity of the Froude number $F_{r}$ and the sedimentation parameter $\gamma$ in the box model}

In this paper the Froude number $F_{r}$ was set at 1.0, and we found that a good fit to six of the eight $\mathrm{V}$-shaped-valley experiments was achieved with sedimentation parameter $\gamma=0.7$. The exception was experiment 7 where we used $\gamma=0.5$. No attempt was made to find the best choice of parameters for each experiment because the agreement between the model and the experimental results was already reasonably good.

To show the insensitivity of our results to the values of $F_{r}$ and $\gamma$ we ran calculations in which we varied $F_{r}$ by $\pm 10 \%$, and $\gamma$ by $\pm 14 \%$. The effect of varying $F_{r}$ and $\gamma$ on the box model mass deposition occurs through the ratio $F_{r} / \gamma$ which, in the flat-bottomed valley, occurs as a factor $\left(F_{r} / \gamma\right)^{2 / 5}$ in $X_{R F}$. Changes of $25 \%$ in this ratio therefore result in changes to $X_{R F}$ of $\sim 10 \%$. The estimate of $X_{R V}$ for the V-shaped valley when $h_{0} \leqslant a$ involves the factor $\left(F_{r} / \gamma\right)^{4 / 7}$ in $X_{R V}$. In this case, changes of $25 \%$ in this ratio therefore result in changes to $X_{R V}$ of $\sim 14 \%$.

The variation of the $X, t$ curve with changes in $F_{r}$ and $\gamma$ is more complicated though we expect it will be dominated by the factor $F_{r}$ in (4.2). The complication comes about because the mass deposit depends on $\gamma$ and this affects $g^{\prime}$. To estimate the sensitivity of the $X, t$ curve to these changes we ran box model calculations for experiment 5. These included a set with $F_{r}=1$ and $\gamma=0,0.7,0.8$, and a set with $\gamma=0.7$ and $F_{r}=0.9,1.0,1.1$.

The results are shown in figure 16 where it is clear that the changes to the $X, t$ curves are small when $F_{r}$ is fixed and $t \lesssim 40 \mathrm{~s}$, but steadily increase until they reach $\sim 10 \%$ at $t \sim 90 \mathrm{~s}$. When $\gamma$ is fixed and $F_{r}$ changed there is a noticeable deviation 
between the curves for $t \gtrsim 15 \mathrm{~s}$. This fractional deviation is approximately equal to the fractional change in $F_{r}$.

\section{REFERENCES}

Altinakar, M. S., Graf, W. H. \& Hopfinger, E. J. 1996 Flow structure in turbidity currents. J. Hydraul. Res. 34, 713-718.

Batchelor, G. K. 1967 An Introduction to Fluid Dynamics. Cambridge University Press.

Bonnecaze, R. T., Huppert, H. E. \& Lister, J. R. 1993 Particle-driven gravity currents. J. Fluid Mech. 250, 339-369.

BRitter, R. E. \& Linden, P. F. 1980 The motion of the front of a gravity current travelling down an incline. J. Fluid Mech. 99, 531-541.

Cantero, M. I., Balachandar, S., Garcia, M. H. \& Bock, D. 2008 Turbulent structures in planar gravity currents and their influence on the flow dynamics. J. Geophys. Res. 113, C08018, doi:10.1029/2007JC004645.

DAde, W. B. \& Huppert, H. E. 1995a A box model for non-entraining, suspension-driven gravity surges on horizontal surfaces. Sedimentology 42, 453-471.

DADE, W. B. \& HupPert, H. E. $1995 b$ Runout and fine-sediment deposits of axisymmetrical turbidity currents. J. Geophys. Res. 100, 18597-18609.

De Roois, F. \& Dalziel, S. B. 2001 Time-and space-resolved measurements of deposition under turbidity currents. Spec. Publ. Intl Assoc. Sediment 1, 207-215.

Garcia, M. H. 1994 Depositional turbidity currents laded with poorly sorted sediment. J. Hydraul. Engng 120, 1240-1263.

Hacker, G., Linden, P. F. \& Dalziel, S. B. 1996 Mixing in lock-release gravity currents. Dyn. Atmos. Oceans 24, 183-195.

Hallworth, M. A., Huppert, H. E., Phillips, J. C. \& Sparks, R. S. J. 1996 Entrainment into two-dimensional and axisymmetric turbulent gravity currents. J. Fluid Mech. 308, 289-311.

Huppert, H. E. 1998 Quantitative modelling of granular suspension flows. Phil. Trans. R. Soc. 356, 2471-2496.

Huppert, H. E. \& Simpson, J. E. 1980 Quantitative modelling of granular suspension flows. J. Fluid Mech. 99, 785-799.

Kneller, B. C., Bennett, S. J. \& McCaffey, W. D. 1997 Velocity and turbulence structure of density currents and internal solitary waves: potential sediment transport and the formation of wave ripples in deep water. Sedimentary Geol. 112, 235-250.

KNeller, B. \& BuCKeE, C. 2000 The structure and fluid mechanics of turbidity currents: a review of some recent studies and their geological implication. Sedimentology 47 (Suppl. 1), 62-94.

Martin, D. \& Nokes, R. 1988 A fluid-dynamical study of crystal settling in convecting magmas. Nature 332, 534-536.

Martin, D. \& Nokes, R. 1989 A fluid-dynamical study of crystal settling in convecting magmas. J. Petrol. 30, 1471-1500.

Mohrig, D. \& Buttles, J. 2007 Deep turbidity currents in shallow channels. Geology 35, 155-158.

Monaghan, J. J., Cas, R. F., Kos, A. \& Hallworth, M. 1999 Gravity currents descending a ramp into a stratified tank. J. Fluid Mech. 148, 227.

Monaghan, J. J., Mériaux, C. A., Huppert, H. E. \& Monaghan, J. M. 2009 High Reynolds number gravity currents along V-shaped valleys. Revised for Eur. J. Mech. B/Fluids.

RotTman, J. W. \& Simpson, J. E. 1983 Gravity currents produced by instantaneous releases of a heavy fluid into a rectangular channel. J. Fluid Mech. 135, 95-110.

RotTman, J. W., Simpson, J. E. \& Hunt, J. C. R. 1985 Unsteady gravity current flows over obstacles: some observations and analysis related to the Phase II trials. J. Hazardous Mater. 11, 325-340.

Segrè, P. N., Liu, F., Umbanhowar, P. \& Weltz, D. A. 2001 An effective gravitational temperature for sedimentation. Nature 409, 594-597.

Simpson, J. E. 1997 Gravity Currents: In the environment and the Laboratory. Cambridge University Press.

TAKAGI, D. \& Huppert, H. E. 2007 The effect of confining boundaries on viscous gravity currents. J. Fluid Mech. 577, 495-505.

TAKAGI, D. \& HuPPERT, H. E. 2008 Viscous gravity currents inside confining channels and fractures. Phys. Fluids. 20(2), 023104. 